\title{
They have needs, they have goals: using communication theories to explain health-related social media use and health behavior change
}

\begin{abstract}
Today many people use social media sites such as Facebook, Twitter and YouTube. Despite extended research on the use of social media for health-related issues, little research attention has been devoted to understanding the goals and needs behind such use and its effects on health behavior. The goal of this study is to fill this research gap by employing Uses and Gratifications theory and Media System Dependency theory. The data for this study were collected through six focus groups of internet users in Israel. The findings show that people utilize a number of social media sites for health purposes, according to specific needs and goals. These needs and goals motivate individuals to choose timing, search language and search topics and to establish criteria for assessing the quality of information and its publishers. The social media is perceived to influence health behavior on different levels.
\end{abstract}

Volume 6 Issue 2 - 2017

\section{Dennis Rosenberg, Rita Mano, Gustavo S Mesch}

Associate Professor, Department of Human Services, University of Haifa, Israel

Correspondence: Rita Mano, Associate Professor Department of Human Services, University of Haifa, Israel,Tel +9725476 I 1608, Fax +97248249282,

Email ritamano@research.haifa.ac.il

Received: April 25, 2017| Published: June 20, 2017

Keywords: social media, facebook, twitter, youtube, health-related issues, health behavior, gratification, communication theories, internet, health habits

\section{Introduction}

During the last two decades, the Internet has become one of the dominant sources of health information, mainly due to facilitated access $^{1}$ and the possibility of retrieving relevant health information. ${ }^{2}$ Among other things, health information was found to increase health literacy, ${ }^{3,4}$ help individuals develop intentions to change health habits and enable them to carry out these changes. ${ }^{1}$

Over the past tenyears, the use of social media sites has risen constantly. ${ }^{6,7}$ Among these sites are content communities such as YouTube $^{6}$ and social networking sites such as Facebook. ${ }^{4,7}$ These sites are characteristic of the Web 2.0 concept, which emphasizes interaction and collaboration between users. ${ }^{6,7}$

Academic research has made several theoretical distinctions between social media sites. First, Kaplan \& Haenlein ${ }^{6}$ made a general distinction between two dimensions: self-presentation/disclosure and social presence/media richness. The first dimension refers to providing personal information according to the self-impression one desires to cultivate on social media. It may be categorized as high or low. The second dimension refers to the degree of possible contact with others (social presence) and to the amount of information exchanged within a specific time period (media richness). Its degree can be low, medium or high. ${ }^{7}$ Thus, for example, Facebook is characterized by a medium level of social presence/media richness and a high level of self-disclosure. YouTube is also marked by a medium level of social presence/media richness, but a low level of self-disclosure. Househ et al., ${ }^{8}$ point to another distinction between social media sites: type of content provided. YouTube and Instagram provide visual content (pictures, videos), whereas the content on Facebook, forums or Twitter is primarily textual.

Beginning in 2010, social media research began focusing on health aspects. A study by Murthy et al., ${ }^{9}$ investigated cancer networks activity on Twitter. Newman et al., ${ }^{10}$ studied patterns of health information sharing on Facebook. Thackeray, et al., ${ }^{11}$ sought to explain health-related use of social networking sites. Vaterlaus et al., ${ }^{12}$ studied the perceived influence of social media use on health habits. Yet none of these studies explained the needs and goals that motivate individuals to use social media for health. In addition, most tended to examine only one specific site (whether Facebook, Twitter or some other site) or only one group on these sites, as in case of Zhang et al., ${ }^{13}$ Moreover, even though the study by Vaterlaus et al., ${ }^{12}$ is relatively similar to the current study, it was conducted among young users only.

The current study seeks to fill these gaps using the Users and Gratifications (U\&G) and Media System Dependency (MSD) theories to explain both the health-related use of social media and the influence of this use on health behavior. By doing so, this study will contribute to the existing literature in several ways. First, it will refer to use of various social media sites and not to one particular site. Second, it will contribute to understanding the needs and goals of health-related use of social media from the communication perspective. Third, it will contribute to knowledge concerning the influence of social media on health behavior among the entire population, regardless of age, gender and ethnic origin.

Therefore, the research questions are as follows:

RQ1: How do individuals use social media for health purposes? What are the goals and needs motivating them to such use?

RQ2: How are these goals and needs expressed in the healthrelated use of social media?

RQ3: How does the health-related use of social media affect health behavior?

\section{Literature review}

Communication theories explain the reasons and motives that lead individuals to use a particular source of information. According to the U\&G theory, ${ }^{14,15}$ individuals have various personal needs, and they will use source(s) of information to gratify these needs. ${ }^{15}$ 
The theory outlines four basic categories of needs: information, entertainment, social interaction and personal identity. ${ }^{14}$ According to MSD theory, ${ }^{16-18}$ individuals will turn to specific source(s) in the face of ambiguity, uncertainty or threat. ${ }^{19}$ The theory outlines six goals that individuals seek to meet using a particular medium in order to reduce uncertainty or threat: self-understanding, social understanding, action orientation, interaction orientation, solitary play and social play. ${ }^{16,20}$ While meeting these goals, individuals may also become dependent on the medium. Such dependency is established when the source provides unique information tailored to individuals' goals. ${ }^{17}$ Such cases may lead to increased exposure to the content provided by a source. ${ }^{16}$ After being so exposed, individuals may change their beliefs, values and/or behavior. ${ }^{17}$

Both U\&G theory ${ }^{14,16}$ and MSD theory ${ }^{21}$ can explain general social media use. Yet no existing research explains health-related use of social media using these theories. This study seeks to fill this gap. After a brief discussion of how social media and health are interrelated, we discuss the influence of technology on health.

\section{Social media and health}

Like the Internet as a whole, social media is used both for searching for health information and for communicating regarding health issues. However, social media platforms are still not the first option in searching for health information because they are considered suitable mostly for entertainment and leisure activities. ${ }^{22}$ Only about one percent of health inquiries begin on social media sites. ${ }^{23}$ Nevertheless, according to Woolley et al., ${ }^{4} 39 \%$ of U.S. internet users who searched for online health information did soon social networking sites. Sharma \& Kaur $^{24}$ mention similar data regarding Internet users in the AsiaPacific region. There is also evidence of growing use of Facebook and YouTube by health institutions. ${ }^{25}$

In the past severalyears, many health-related social media sites, such as PATIENTSLIKEME" or CAMONI ("Similar to me", "Like me" in Hebrew) $)^{26}$ have been established. In addition, health-related groups with hundreds and sometimes thousands of participants have been established on Facebook, Twitter ${ }^{9,27}$ and dedicated forums. ${ }^{28}$ In these online spaces, individuals contact others who have or had similar health issues. ${ }^{27}$

\section{Technology and health behavior changes}

The health-related use of technology itself is not the last stop when discussing personal healthcare. This use, as previously mentioned, may influence health behavior as well, that is, any behaviors that can lead to either improving or worsening a health condition. ${ }^{29}$ Health behavior changes may include adopting new health behavior, correcting existing behavior or adopting a combined behavior that includes elements both of existing and corrected behaviors. ${ }^{30}$

The use of technology is said to influence individuals' health behavior since it is capable of shaping their social norms. ${ }^{13}$ Similarly, health-related use of technology may affect health behavior. For example, online health information may cause individuals to change their perceptions about health ${ }^{31}$ and to adopt positive health behaviors. ${ }^{2}$ Several studies support these claims regarding the healthrelated Internet use. Mano et al., ${ }^{1}$ found that the search for online health information affects various types of health behavior changes. McKinley \& Wright ${ }^{5}$ found that frequency of search for online health information was positively associated with healthy eating intentions. Social media is also claimed to influence health behavior ${ }^{32}$ for example regarding food choices - due to their interactive nature, including reading other people's posts. ${ }^{12}$
In this study, we examine how individuals use different social media for health purposes and how this use influences their health behavior. After describing the study methodology, we present the findings in a separate section and summarize them in the discussion section.

\section{Methods}

This study is a part of a larger project funded by the Israel Science Foundation. The main goal of this project is to assess the effect of health-related social media use on health behavior. The stage of the project described in this study employed six focus groups and served as a pilot for the quantitative part of the project. Focus groups provide a large amount of rich and in-depth data compared to other (qualitative) methods. ${ }^{33}$ Since health-related use of social media has barely been studied in Israel, except for the research by Magnezi et al., ${ }^{26}$ it was important to gather abundant data using an appropriate method.

\section{Instruments}

The study used two questionnaires: one for recruitment and the other for the study itself. The recruitment questionnaire included 12 items:

i. Five socio-demographic attributes (gender, age (age group and exact age - two separate items), nationality, country of birth, place of residence),

ii. One item on social media use,

iii. Two items representing health evaluation (perceived health status and presence of diagnosed health conditions) and

iv. One item (divided in two) regarding health-related activity (search for health information online and participation in health discussions).

In the last two items participants were asked

i. Whether they would be willing to take part in focus groups and

ii. If yes, to provide their full name and telephone number to arrange a date for the session.

The respondents were screened and assigned to groups according socio-economic attributes (except for gender) and health-related activity on the Net.

The study questionnaire was used to ensure that all the topics were covered. ${ }^{34}$ In this paper, we describe only those relevant to this study. The first topic dealt with the search for health information on social media. The participants were asked about the social media sites they use for this purpose, the reasons for this use, the characteristics of their search (topics, language and timing of search), and their criteria for identifying credible health information on social media and credible publishers. The second topic concerned their active participation on social media. The participants were asked about the groups in which they participate, the reasons for their participation, the extent of their activity and whether they subscribe to pages. With respect to the third issue, health behavior changes, the participants were asked to discuss the role of social media use in those changes.

\section{Participants}

The study population included 57 adults from both the Jewish and the Arab sectors in Israel. The participants in the Jewish sector were recruited by the Panels Research Center via its online survey panel. In the Arab sector, one person who was experienced in conducting 
focus groups recruited the participants and led the group. Participants were divided into six groups. In the Jewish sector, two of the groups consisted of individuals residing in the central part of Israel:

a. Younger (ages 22-44) and

b. Older (ages 45-65) participants.

Three additional groups comprised

c. Immigrants from the former Soviet Union,

d. Residents of the northern part of Israel and

e. Residents of the southern part of Israel. In each of these three groups, participants ranged in age from 22 to 65 . The groups in the Jewish sector comprised both genders, while those in the Arabic-speaking group were all females between the ages of 24 and 45 and resided mainly in Nazareth.

\section{Design}

The group interviews were conducted using a semi-structural grid questionnaire.$^{34}$ Each session was recorded and transcribed verbatim. During the transcription, no personal data were recorded. ${ }^{12}$ Each session referred to the same topics discussed in same order. ${ }^{31}$

\section{Procedure}

All group sessions took place in June 2015. At the beginning of each session, the moderator welcomed the participants, explained the purpose of the study to them and set the rules for the discussions. The moderator also advised them that the session was being audio recorded. No participant expressed an objection to that. Each session lasted 90 minutes. At the end of each session, participants received compensation for their participation (150 NIS by gift card).

\section{Results}

First, we describe the results in response to the first research question dealing with the needs and goals that motivate participants to use social media for health-related purposes. The findings refer both to the search for health information and to active participation on social media.

\section{Search for health information on social media}

In this section, we describe the health-related use of each site mentioned in the focus groups. At the end of each description, we outline the main needs and goals of use.

Camoni: The Israeli health-related social networking site: This community is highly valued by its members on several levels. First, it provides technical information about coping with diseases/problems:

"I have some medical problems and I search (on Camoni) for how other people, not specifically doctors, cope with it... It is more like interpersonal communication, not that I search for specific medical information, I search for how people cope with such issues" (Immigrants).

Second, Camoni serves as a support group in which people not only seek information but also provide it to others (not only patients):

"This is a network that supports not only patients themselves but also members of their families..." (South)

As can be seen, Camoni use derives from two needs: need for information and need for social interaction. The goals are mainly selfunderstanding (observing how others cope with similar problems) and interaction orientation (approaching others to get/provide advice and/ or support).

Forums: Are one of the most popular formats for searching for health information. Users can read replies to questions posted by other users or ask questions themselves. Although in the past forums were very popular, today it seems they are mainly used for occasional queries posed by those facing some problem for the first time:

"Forums have lost their functionality today. People automatically go to Facebook" (North).

Thus, the main need gratified by visiting forums is in information. The main goal is self-understanding.

Face book: is a very popular platform in terms of health-related use. This use is marked by three characteristics:

A. User's private territory. During interactions between participants and their friends, health issues are rarely discussed. The feeling is that the platform is not private enough to provide personal health information and does not incorporate experienced persons who can relate to serious health issues:

"...I relate to it as a social place, and as such I do not have a reason to search for information there" (Center, 45-65).

Therefore, similar to Newman et al., ${ }^{10}$ study, participants are very concerned about the personal identity they cultivate on Facebook:

"I don't want everyone who is my friend on Facebook to know my problems - not general questions such as sports but more like medical ones" (Immigrants).

"Facebook is so open to others. If I 'like' a specific topic, everybody will know it. And there is no need for them to know that I read about or am interested in particular things. This is something private that belongs to me" (Arab sector women).

Therefore, the main issues raised on the Facebook platform related to physical activity or nutrition - areas that do not require specialization in health or medical fields.

A. "Liking" pages. Participants tend to "like" the pages of health professionals or institutions (dentists, health institutes, fitness club, dieticians). "Liking" represents temporary interest in a page. As a result, many participants remember that they "liked" a page but do not remember any interaction with the relevant page over time. This is mostly because the benefits of "liking" pages are not immediate:

“... I examine all the pages I visit, but if a specific page makes some positive impression according to criteria I define, then I will 'like' it”' (North).

“... I 'like' particular pages that I think will benefit me” (Arab sector women).

B. Connection to groups that deal with diseases, problems or conditions relevant to the visitor. In such groups, members raise issues for discussion that are related to diseases or conditions, make recommendations and support each other:

"There are groups of dieticians and fitness groups, so in my opinion, this is the best source, because I also have friends on Facebook who 
train using specific methods and they also make recommendations and you can also compare the results. Examples are healthier foods or those more suitable for those who train, recommendations about proteins and stuff like that, all about chemistry and everything related. In my opinion, Facebook is an excellent platform" (Immigrants).

To summarize, Facebook serves the need for information, social interaction and personal identity. The main goals are selfunderstanding, action orientation and interaction orientation.

YouTube: is a less used site for searching for health information. In most cases, participants do not intentionally visit the site, but rather are redirected to its videos by Google searches. If they search on YouTube itself, their search usually concerns lifestyle:

"I wanted to improve my swimming, so there are some suggestions there." (Center, 45-65).

"I study fitness using YouTube, do fitness. If I want to improve skills in my group, I visit YouTube and learn it well" (North).

YouTube has the advantage of being illustrative. Therefore, it creates additional value for those searching for an online instructor:

"On YouTube there is a very interesting explanation about how a colonoscopy is performed. You do not need to read. Reading also involves using the eyes, but here you actually see what is going on. It is like what is preferable: reading a book or watching a video? The video gives a short summary" (Center, 45-65).

Twitter/Instagram: These two platforms are rarely used to search for health information. The younger participants mentioned short videos about sports exercises that are uploaded to Twitter:

"There is really this stuff that people talk (on Twitter) that they've started to lead a healthier lifestyle. As known, the number of characters is restricted, so they write a few words and give Facebook links and places where this can be seen. However, many people write about themselves, either about their successes or about their intentions in health issues. People want to bolster themselves and their exterior, and they can write it shorter and faster on Twitter" (North).

The visual element is very important to Instagram users:

"From the visual point of view and leading a healthy lifestyle, it (Instagram) is a very strong network to do that, because you see people running, eating, 10-second clips." (North).

Thus, YouTube, Twitter and Instagram gratify a need for visual information. The main goal relates to self-understanding.

\section{Active participation on social media}

Online health groups can be characterized along several axes:

A. Purpose: task-oriented vs. random topic groups. The first type is established based on health issues. The second type is initially established based on a non-health issue, but sometimes deals with health-related issues (like "Mama-zone").

B. Openness: open vs closed groups. Open groups allow everyone to join. In the case of closed groups, new participants can join only after authentication by a group founder/administrator.

C. Degree of centralization: Centralized vs decentralized groups. Centralized groups have a leader who defines the topics for discussion, raises issues and answers the questions. In decentralized, the members consult each other and raise questions and answers.

People participate in these groups mainly to help others and be helped in the future (need for information and for social interaction):

"If I have something that can help somebody else it is interesting and good to contribute. I also accumulate the information for myself because if something like this happens to my child, I will know what to do" (South).

The participants mentioned that for the most part they are passive in these groups. This because their dominant need is for information.

\section{Characteristics of use}

Now, we present the results pertaining to the second research question. The following topics were mentioned as issues of interest while searching on social media: surgery, fitness and nutrition, and pregnancy and motherhood. In line with the need for information and the goal of self-understanding, the participants wanted to know more in order to decrease their degree of fear, to gain perspective and to improve their actions.

Regarding language, most searches are carried out in Hebrew and fewer in other languages. Regarding the use of Hebrew, the need for exact and relevant information drives participants to prefer Hebrew over other languages. This emphasis on Hebrew was especially pronounced among Arab-speaking female participants.

"We live in a place where all the medical institutions are in Hebrew. And if I search in Arabic, I get information from the Gulf countries, from Egypt, and this is not my destination. I want to search here more, because when I need to go to the doctor, I'll know where to go".

Regarding the timing of searching for health information on social media, the participants search both before and after visiting their health professionals (HPs), similar to the results of a U.S. study. ${ }^{31}$ Younger participants prepare themselves less than older ones. Young participants search in order to decide whether "it is worth consulting HP" or whether "I can solve it myself":

"Suppose I have some injury, is it worth going to the doctor or not, yes/no; is it something serious or am I worrying myself for no reason; stuff like this. If it is another issue, let's suppose breast feeding or other health matters, so I won't go to the doctor at all, I prefer forums" (Center, 22-44).

In contrast, older participants prefer to come to HP more prepared:

"I noticed that if you come with something, and something that has some basis, not simply rumors, the doctor has more patience for you... either he is afraid of giving you inaccurate information or he has more respect for you, or a combination of both. I don't care which it is but I always come with information" (Center, 45-65).

After consulting HP, participants regardless of age visit social media to validate information. This finding is similar to the findings of a Swiss study. ${ }^{34}$

"I want to get an opinion about the treatment... Even if it is antibiotics, I want to read about antibiotics before I take it..." (Center, 22-44).

"After he prescribed a medication, I checked what the side effects are” (Immigrants). 
Concerning the quality of information on social media, the amount of available information sometimes creates a sense of overload and confusion. Nevertheless, the participants were able to identify credible information on social media using several criteria:

\section{i. Similarity of replies given to same questions:}

"The crowd method. When you see the same answer several times. Or the same condition with the same recommendations. You understand that this is probably the right answer" (South).

\section{ii. Publisher's identity:}

"If this information on social networking sites originates from the site of Ministry of Health/HMO, so okay, I can rely on it. But if this is something private, as somebody here said a dietician, and we don't know each other, you will still have doubts and suspicion" (Arab sector women).

\section{iii. Visibility effect:}

"It is accompanied by a video... and when you see it in front of your eyes it seems a little bit more credible, because you see that the person has really done that and it succeeded, so it is more credible..." (Center, 22-44).

"It seems more credible to me, not simply a forum where somebody wrote and recommended, but somebody who made a video or explanation about it..." (Center, 45-65).

In addition, participants use two criteria to identify the publishers of accurate and reliable information:

\section{i. Profile picture:}

"The advantage of this site is that you also can see the picture of the doctor who answers you. This shows credibility" (Center, 22-44).

\section{ii. Personal familiarity:}

\section{"A familiar person is more credible" (Center, 22-44).}

Thus, the need for (high quality) information was the main motivator for establishing criteria for credibility of information and of information publishers.

\section{Health behavior changes}

In this subsection, we present the results pertaining to the third research question dealing with participants' perceptions of the influence of health-related social media use on health behavior. Social media contribute to health behavior changes both by clarifying the dangers resulting from not making the change and by demonstrating the effectiveness of adopting the change. The dangers and risks are better presented on textual platforms (forums, Facebook), while the effectiveness is better transmitted visually (Instagram, YouTube):

"I have a good friend who is a TRX instructor, and he regularly uploads training sessions in the park. If I was not like I am today, I would be in the park today. If I did not know what the training is like and at the same time was seeing the picture, there is no doubt I would call him and ask him what to do if I was interested. If I didn't have Facebook I wouldn't know this existed, but since I've seen it, it is more interesting for me" (Center, 22-44).

In conclusion, participants can be divided into three groups according to how they perceived the influence of social media on their health behavior changes:

A. Those who were not influenced at all:

"No, a medical check" (South).

"No, it is related to self-consciousness" (Center, 22-44).

B. Those who were partially influenced (in addition to other sources):

"Not only from social networks. The doctor I go to said that I should reduce..." (South).

C. Those who were influenced greatly:

"Yes, it was the forums" (South).

"On the milk issue, yes" (Center, 45-65).

\section{Discussion}

The goal of this study was to examine the health-related use of social media and the impact of this use on health behavior. For this purpose, a number of focus groups were interviewed. The study's findings mostly support the assumptions of the theories employed for this study.

\section{Responses to the research questions}

Regarding the first research question, the most dominant need found was the need for information. People search for different kinds of health information, discuss it, share health-related content and more. They do this mostly in order to reduce their fears of specific health problems and to learn how to treat and cope with these problems. Different social media sites provide different types of content and are used to gratify needs for those types. Specifically, YouTube and Instagram gratify the need for visual information, whereas Facebook, forums and Camoni gratify the need for textual information. Two conclusions can be drawn from this finding based on U\&G theory. First, this emphasizes individuals' purposefulness and selectiveness in choosing their information channel. ${ }^{14}$ Second, several sources are able to gratify one need (and, as can be seen, several needs can be gratified by using one source). The need for social interaction is less pronounced. In this context, participants use social media for contacting similar others to both elicit and provide social support, which can improve health. ${ }^{35}$ An additional need mentioned is the need for personal identity. Participants were reluctant to discuss their problems on open platforms such as Facebook due to the desire to make a good impression. Based upon the terminology proposed by Goffman et al., ${ }^{36}$ the identity intended to impress others came to frontstage, while the real identity was relegated backstage. Participants did not express the need for entertainment at all.

Regarding MSD theory, the main goal of health-related use of social media was self-understanding. Participants wanted to know (how to do) different things relevant to their health problems and how others cope with similar problems. Facebook, Camoni and forums seem to be helpful sources for achieving this goal. Another pronounced goal was interaction orientation. Participants mentioned that several social media sites helped them approach people with similar experience. As in Morton \& Duck, ${ }^{17}$ the goals of play were not mentioned. To summarize, social media sites serve several goals that individuals seek to meet in order to reduce threat or uncertainty, ${ }^{19}$ both of which are caused by their own health condition or that of someone from their social circle. In accordance with MSD theory, these goals 
can be met through a single source. ${ }^{18}$ Conversely, one goal (like selfunderstanding) can be met through several sources.

With respect to the second research question, different goals and needs motivate participants to establish a strategy of health-related social media use. The need for exact and credible information that is relevant to the Israeli reality and the goal of self-understanding motivated them to search mainly in Hebrew, both before and after visiting HP. This need also led them to establish criteria for evaluating whether the information and those who publish it are reliable and trustworthy. This strategy helps them obtain information/support efficiently and use it effectively for their health.

Concerning the third research question, health-related use of social media indeed influences whether people make health behavior changes, thus supporting the MSD theory. It seems that making such changes depends on the extent to which social media are perceived as helpful in satisfying the goals. For example, those who reported not being influenced by social media in their health behavior may not perceive social media as a helpful source, or may perceive the extent of its helpfulness as limited. In contrast, those who reported being influenced may perceive social media as being extremely helpful.

\section{Limitations and recommendation}

The study has two main limitations. First, it does not allow us to generalize the results due to its qualitative nature. Future studies should use quantitative methods to examine associations between health-related social media use and health outcomes. Second, not all population groups were represented. For example, there were no Arabic-speaking men among the participants. It is also possible that there will be differences between individuals from the same ethnic category but living in different regions of Israel in terms of provided information. Nevertheless, this study provides insight into the use of health-related social media and outlines the main needs and goals driving this use. Future studies are warranted to continue this trend and provide a more generalizable picture of this phenomenon.

\section{Acknowledgements}

We would like to thank Panels Research Institute for their assistance and cooperation.

\section{Conflict of interest}

Author declares that there is no conflict of interest.

\section{References}

1. Mano R. Online health information, situational effects and health changes among E-patients in Israel: A “push/pull” perspective. Health Expectations. 2014;18(6):2489-2500.

2. Xiao N, Sharman R, Rao HR, et al. Factors influencing online health information search: An empirical analysis of a national cancer-related Survey. Decision Support Systems. 2014;57:417-427.

3. Mano R. Social media and online health services: A Health Empowerment perspective to online health information. Computers in Human Behavior. 2014;39:404-412.

4. Woolley P, Peterson M. Efficacy of a health-related Facebook social networking site on health-seeking behaviors. Social Marketing Quarterly. 2012;18(1):29-39.

5. McKinley C, Wright PJ. Informational social support and online health information seeking: Examining the association between factors contributing to healthy eating behavior. Computers in Human Behavior. 2014;37:107-116.
6. Kaplan AM, Haenlein M. Users of the world, unite! The challenges and opportunities of social media. Business Horizons. 2010;53:59-68.

7. Merolli M, Gray K, Martin-Sanchez F. Health outcomes and related effects of using social media in chronic disease management: A literature review and analysis of affordances. J Biomed Inform. 2013;46:957-969.

8. Househ M, Borycki, E, Kushniruk A. Empowering Patients through Social Media: The Benefits and Challenges. Health Informatics J. 2014;20(1):50-58

9. Murthy D, Gross A, Oliveira D. Understanding cancer-based networks in Twitter using Social Network Analysis. In Semantic Computing (ICSC). 2011 Fifth IEEE International Conference. 2011. p. 559-566.

10. Newman MW, Lauterbach D, Munson SA, et al. It's not that I don't have problems, I'm just not putting them on Facebook: Challenges and opportunities in using online social networks for health. In Proceedings of the ACM 2011 Conference on Computer-Supported Cooperative Work. 2011. p. 341-350.

11. Thackeray R, Crookston BT, West JH. Correlates of health-related social media use among adults. J Med Internet Res. 2013;15(1):e21.

12. Vaterlaus JM, Patten EV, Roche C, et al. Gettinghealthy: The perceived influence of social media on young adult health behaviors. Computers in Human Behavior. 2015;45:151-157.

13. Zhang Y, He D, Sang Y. Facebook as a platform for health information and communication: A case study of a diabetes group. $J$ Med Syst. 2013;37:9942-9953.

14. Brandtzaeg PB, Heim J. Why people use social networking sites. In International Conference on Online Communities and Social Computing. 2009. p. 143-152.

15. Smock AD, Ellison NB, Lample C, et al. Facebook as a toolkit: A Uses and Gratification approach to unbunding feature use. Computers in Human Behavior. 2011;27(6):2322-2329.

16. Loges WE. Canaries in the coal mine: Perceptions of threat and Media System Dependency relations. Communication Research. 1994;21(1):5-23.

17. Morton TA, Duck JM. Communication and health beliefs: Mass and interpersonal influences on perceptions of risk to self and others. Communication Research. 2001;28(5):602-626.

18. Patwardhan P, Yang J. Internet Dependency Relations and online consumer behavior: A Media System Dependency theory perspective on why people shop, chat and read news online. Journal of Interactive Advertising. 2003;3(2):57-69.

19. Tustin N. The role of patient satisfaction in online health information seeking. J Health Commun. 2010;15(1):3-17.

20. Grant AE, Kendall Guthrie K, Ball-Rokeach SJ. Television shopping: A Media System Dependency perspective. Communication Research. 1991;18(6):773-798.

21. Lee CS. Exploring emotional expressions on YouTube through the lens of Media System Dependency theory. New Media and Society. 2012;14(3):457-475.

22. Zheng Y. Patterns and motivations of young adults' health information acquisitions on Facebook. Journal of Consumer Health on the Internet. 2014;18(2):157-175.

23. Chen W, Lee K-H. More than search? Informational and participatory eHealth behaviors. Computers in Human Behavior. 2014;30:103-109.

24. Sharma P, Kaur PD. Effectiveness of web-based social sensing in health information dissemination - A review. Telematics and Informatics. 2017;34(1):194-219. 
25. Antheunis ML, Tates K, Nieboer TE. Patients' and health professionals' use of social media in health care: Motives, barriers and expectations. Patient Education and Counseling. 2013;92(3):426-431.

26. Magnezi R, Grosberg D, Novikov I, et al. Characteristics of patients seeking health Information online via social health networks versus general Internet sites: A comparative study. Inform Health Soc Care. 2015;40(2):125-138.

27. Greene JA, Choudhry NK, Kilabuk E, et al. Online social networking by patients with diabetes: A qualitative evaluation of communication with Facebook. J Gen Intern Med. 2010;26(3):287-292.

28. Savolainen R. Requesting and providing information in blogs and Internet discussion forums. Journal of Documentation. 2011;67(5):863-886.

29. Sutton S. Health behavior: Psychosocial theories. International Encyclopedia of the Social and Behavioral sciences. 2001:6499-6506.

30. McKinley CJ. Investigating the influence of threat appraisals and social support on healthy eating behavior and drive for thinness. Health Communication. 2009;24(8):735-745.
31. Taha J, Sharit J, Czaja S. Use of and satisfaction with sources of health information among older Internet users and nonusers. Gerontologist. 2009;49(5):663-673.

32. Korda H, Itani Z. Harnessing social media for health promotion and behavior change. Health Promot Pract. 2013;14(1):15-23.

33. Wilkinson S. Focus groups in health research: Exploring the meanings of health and illness. J Health Psychol. 1998;3(3):329-348.

34. Caiata-Zufferey M, Abraham A, Sommerhalder K, et al. Online health information seeking in the context of medical consultation in Switzerland. Qual Health Res. 2010;20(8):1050-1061.

35. Oh HJ, Lauckner C, Boehmer J, et al. Facebooking for health: An examination into the solicitation and effects of health-related social support on social networking sites. Computers in Human Behavior. 2013;29(5):2072-2080.

36. Goffman E. The presentation of self in everyday life. Garden City, NY: Doubleday; 1959. p. 1-17. 